\title{
Karydakis flap: a preferred procedure for sacrococcygeal pilonidal sinus disease (SPNSD).
}

\footnotetext{
1. MBBS, FCPS (Surgery) Assistant Professor

2. MBBS, FCPS (Surgery) Senior Registrar

3. MBBS, FCPS (Surgery) Associate Professor

4. MBBS, FCPS (Surgery) Assistant Professor

5. MBBS, FCPS (Surgery) Assistant Professor

6. MBBS, FCPS (Surgery) Professor Surgery Surgical Unit II

Faisalabad Medical University.
}

Correspondence Address:

Dr. Muhammad Akram

Department of Surgical Unit II

FMU.

akramdr2000@yahoo.com

Article received on:

04/06/2020

Accepted for publication:

07/09/2020
Durr-I-Chaman', Tayyaba Fatima², Muhammad Akram³, Bashir Ahmed ${ }^{4}$, Sajid Rehman ${ }^{5}$, Muhammad Sajid Sheikh ${ }^{6}$

ABSTRACT... Objectives: To evaluate patients with SPNSD who underwent Karydakis Flap procedure with reference to operative time, drain removal time, hospital stay, complications and recurrence rate. Study Design: Observational descriptive. Setting: Surgical Department of Allied Hospital, Faisalabad. Period: September 2009 to January 2018. Material \& Methods: A total of 117 patients underwent Karydakis Flap procedure. Data was collected according to the above said parameters .Patients were followed up for 12 months. Results: The operative time was 35.5 minutes. Average Hospital stay was 2 days. Drain removed at median of 8 days.17 patients (14\%) developed seroma. Wound Infection occurred in 5 patients (4\%). Wound burst opened in 1 patient $(0.8 \%)$.No recurrence was noted $(0 \%)$ in any patient during a follow up of 1 year. Conclusion: Karydakis Flap procedure is a relatively simple and easy technique with minimal chances of recurrence.

Key words: $\quad$ Complications, Karydakis Flap, Recurrence, Sacrococcygeal Pilonidal Sinus Disease.

Article Citation: Durr-I-Chaman, Fatima T, Akram M, Ahmed B, Rehman S, Sajid M. Karydakis Flap: A preferred procedure for Sacrococcygeal Pilonidal Sinus Disease (SPNSD). Professional Med J 2020; 27(11):2284-2288. https://doi.org/10.29309/TPMJ/2020.27.11.5066

\section{INTRODUCTION}

SPNSD also called "Jeep Disease" is a chronic disease mainly noted in young males and army recruits. ${ }^{1,2}$ It represents a significant portion of the patients remedied in surgery clinics worldwide. ${ }^{3}$ A variety of procedures have been described to treat it, but none has proven to be ideal. The most challenging in treatment of SPNSD is to choose the one most appropriate procedure which is easy, takes less time to perform, allows primary wound closure, reduces prolonged hospitalization and is associated with minimum complications or recurrence rate. ${ }^{4}$ As compared to complex flap procedures for SPNSD, Karydakis Flap procedure is an asymmetric excision flap technique that was first described by "Karydakis" in 1973. It has acceptable recurrence rate of $<1 \%$. 5,6 The purpose of current study is to prospectively analyze operation time, Drain removal time, complications and recurrence rate in all patients with SPNSD who underwent Karydakis Flap procedure.

\section{MATERIAL \& METHODS Study Design}

It was a Observational descriptive conducted at Surgical Department of Allied Hospital, Faisalabad. From September 2009 to January 2018. The number of cases was 117. Patients between 15 to 65 years having primary or recurrent SPNSD.

Patients having acute pilonidal abscesses or perianal fistulas presenting as sacrococcygeal sinus Disease were excluded.

\section{Data Collection Procedure}

The study was carried out after approval from hospital's ethical review committee. All the patients were informed about the nature of the procedure and informed consent obtained. Patients were operated in spinal or general anesthesia depending upon their preferences. All patients were given pre operation I.V injection of third generation cephalosporin. Patients operated 
in prone jackknife position with buttocks strapped apart. Before preparing the area, Asymmetrical incision was drawn with indelible pen, making sure that all sinuses are taken in (Figure-1).

Area prepared with $10 \%$ povidone iodine solution. Incision made through epidermis and dermis and subsequently deepened to presacral fascia. Dissection completed with electro cautery ensuring hemostasis (Figure-2).

After excision of this full ellipse of the affected tissue, flap mobilized adequately and brought across midline without tension. Here before closing we washed wound cavity with Normal saline copiously, so any infected hair/debris gets washed away. Suction drain placed. Wound closed in 3 layers then. Deeper layers with vicryl no.1, in interrupted fashion and superficial layer with vicryl 2/0 in continuous stitches (Figure-3).

Skin was approximated with prolene 4/0 interrupted stitches. I.V antibiotics given for 2 days followed by oral antibiotics for 5 days. Drain removed when output was $<20 \mathrm{ml}$. Patients discharged on 2nd post op day mostly and advised weekly visits for 4 weeks (Figure-4).

Afterwards they were followed up telephonically for one year.

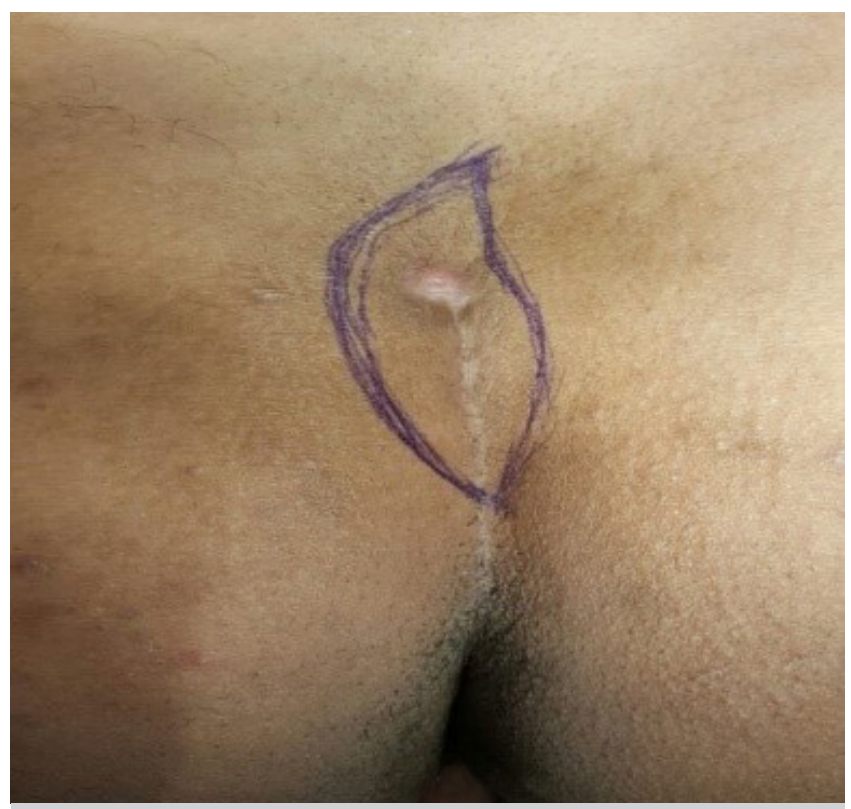

Figure-1. Marking of asymmetrical ellipse.

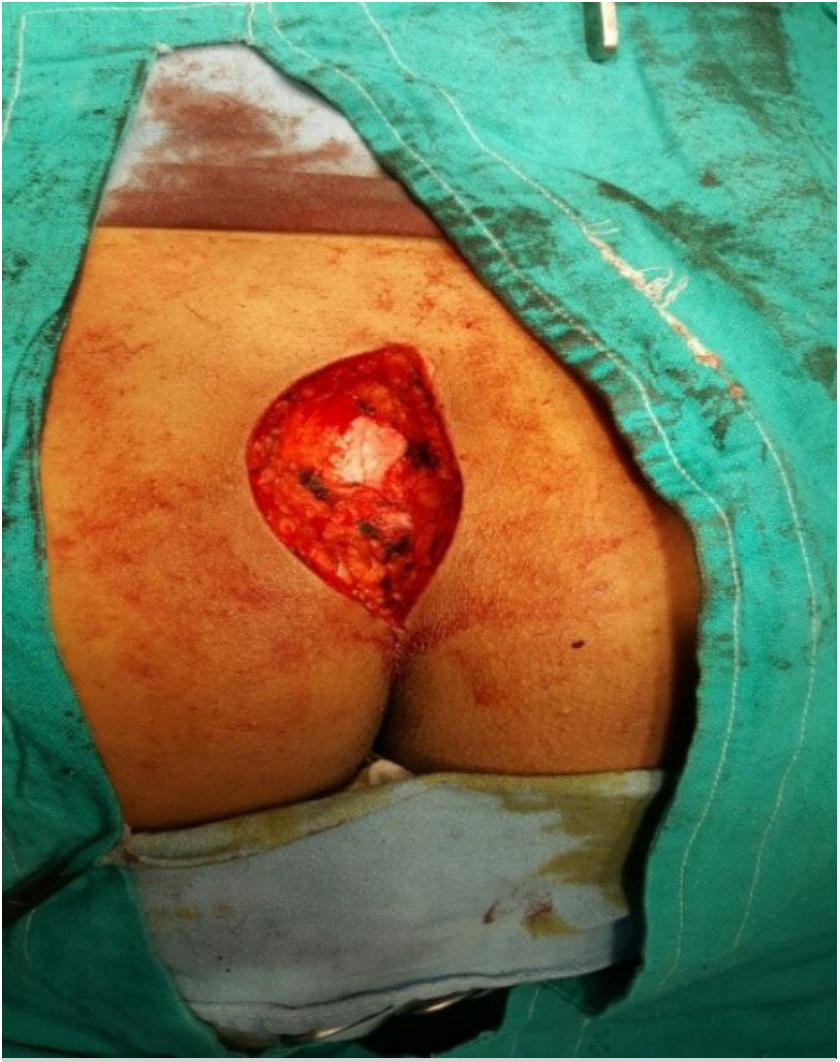

Figure-2. Defect after excision.

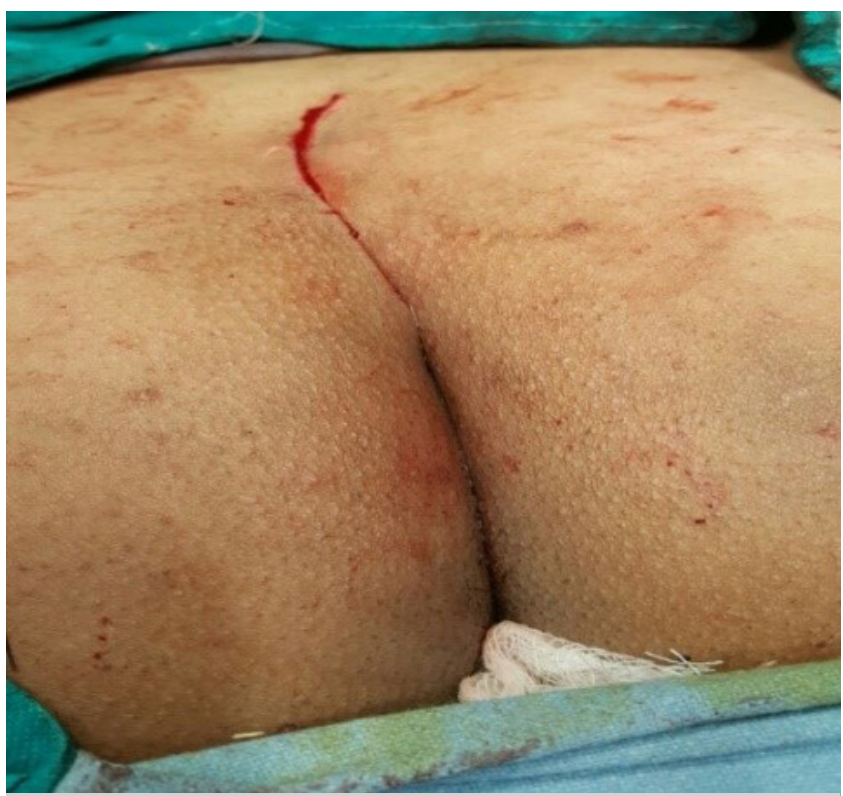

Figure-3. Closure of defect in layered stitches.

An inferior view to show significant midline shift with flattening of natal cleft in another case. 


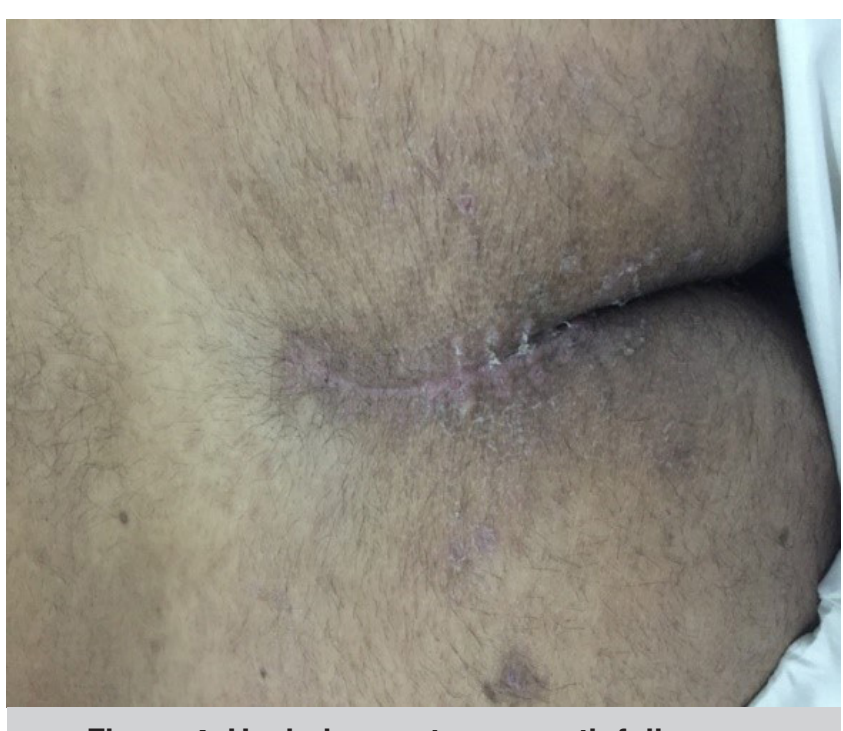

Figure-4. Healed scar at one month follow up.

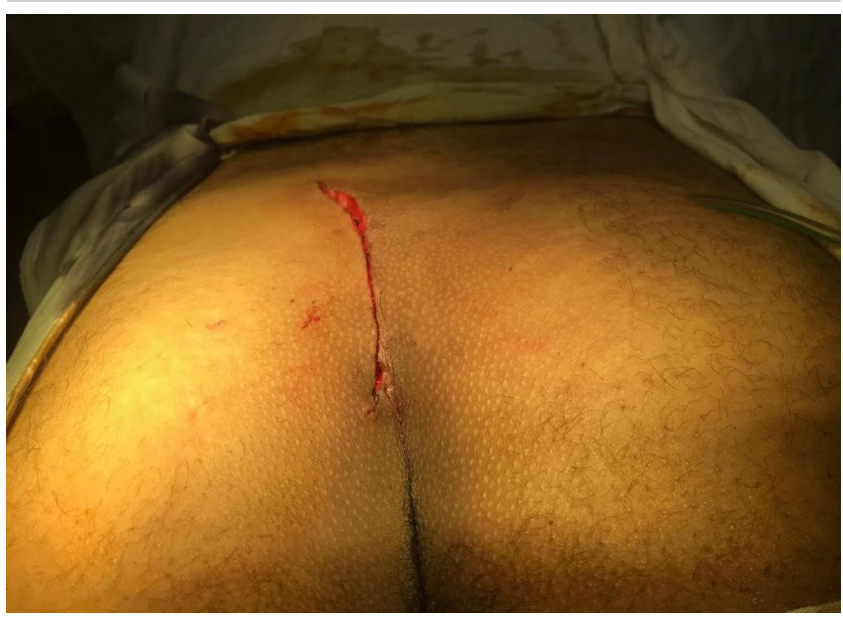

\section{RESULTS}

From Sep 2009 to January 2018, a total of 117 patients underwent Karydakis procedure, out of these 99 were male and 18 were female. Age of patients ranged from $17-47$ years with a mean age of 25.40 years and majority $82 \%$ between 20 35 years age group. The most common symptom was seropurulent discharge in 105 patients, 56 patients had multiple openings.12 patients had a previous surgery and presented with recurrence. The skin was very hairy in 103 patients.

The mean operation time was 35.5 min (range 25-45 min). Hospital stay was 2 days (range 1-4 days). Drain was removed at median of 8 days (range 5-14 days). sutures were removed at $14^{\text {th }}$ day. 17 patients developed seroma after drain removal which was treated with needle aspiration in 5 patients. Wounds were infected which were treated by opening up of the stitches, wound wash and change of dressings in 2 patients. Wounds got burst which was treated by Bactigrass dressings for a period of 3 weeks, wounds healed by secondary intention in these patients. The patients were followed up for a period of 12 months, no recurrence was noted in that period.

\section{DISCUSSION}

SPNSD is a chronic disorder that mainly affects the younger population. Previously it was thought to be congenital but now its aetiology is acquired. Loose hair within sacrococcygeal area due to friction force between buttocks tend to invade the moist natal cleft skin leading to formation of abscess and sinus, so complete excision of the sinuses is necessary to avoid recurrence. ${ }^{7}$ Many surgical procedures have been described in the literature to treat this disease but every technique has its own pros and cons. These procedures can vary from simple wide excision and thus leaving the wound open for secondary healing, to simple excision with midline closure, asymmetrical closure, marsupialization, Bascom repair and different flaps procedures like W plasty, Z plasty, VY plasty, Limberg flap and modified limberg flap. Simple excision is associated with increased morbidity due to delayed healing and is no more a favorite procedure of most surgeons. ${ }^{8}$ Excision with midline closure results in a weak midline scar and is related to increased recurrence rates. Flaps procedures are time consuming and technically more demanding with a risk for flap necrosis and hypoesthesia, though they are related to a low recurrence rate. ${ }^{9}$ On the other hand, Karydakis addresses a lot of such issues. It is simple and easy to perform because it is technically less demanding. ${ }^{3}$ it is associated with low morbidity. it flattens and shifts the natal cleft to one side and thus prevents hair invasion in depth. ${ }^{10,11}$

In this study male to female ratio was $5.4: 1$ and literature supports this preponderance. Mean operative time was 35.5 minutes (range2545 minutes) which is quite less than the other studies. Hospital stay 2 days median (1-4 days) is comparable to other studies. Drain were removed at median of 8 days (range 5-14 days). 
complications like seroma formation following drain removal was noted in $17 \%$, wound infection in $5 \%$, burst wound in $1 \%$ patient. Patients were followed up for a period of twelve months telephonically. All patients were given advice for improving personal hygiene and frequent ablution to prevent recurrence in future. No recurrence was noted in any patients.

The following observations are made during the study that helped in achieving a $0 \%$ recurrence rate:

- Deferring the surgery in case of active infection or abscess formation, till infection is settled after incision drainage or antibiotic course.

- Designing an incision such a way that the lower part of the incision curves upwards away from anal verge helps to reduce contamination.

- After excision washing the cavity copiously with saline to wash off all the loose fat which may be a source of infection.

- Advice about ablution and personal hygiene.

\section{CONCLUSION}

Karydakis is simple and easy technique. it can be performed in less time and it is technically less demanding. It flattens the natal cleft and shift it laterally thus making the scarred area less vulnerable for future recurrence. Moreover, after excision washing off the cavity with saline before closure, post-operative ablution and personal hygiene are important to prevent complications like infection and recurrence.

\section{Copyright@ 07 Sep, 2020.}

\section{REFERENCES}

1. Harlak A, Mentes O, Kilic S, Coskun K, Duman K, Yilmaz F. Sacrococcygeal pilonidal disease: Analysis of previously proposed risk factors. Clinics. 2010; 65(2):125-31.
2. Kapan M, Kapan S, Pekmezci S, Durgun V. Sacrococcygeal pilonidal sinus disease with Limberg flap repair. Techniques in coloproctology. 2002 Apr 1; 6(1):27-32.

3. Yildiz MK, Ozkan E, Odabaşı HM, Kaya B, Eriş C, Abuoğlu $\mathrm{HH}$, Günay E, Fersahoglu MM, Atalay S. Karydakis flap procedure in patients with sacrococcygeal pilonidal sinus disease: Experience of a single centre in Istanbul. The Scientific World Journal. 2013; 2013.

4. Chintapatla S, Safarani N, Kumar S, Haboubi N. Sacrococcygeal pilonidal sinus: Historical review, pathological insight and surgical options. Techniques in coloproctology. 2003 Apr 1; 7(1):3-8.

5. Karydakis GE. New approach to the problem of pilonidal sinus. The Lancet. 1973 Dec 22; 302(7843):1414-5.

6. Kumar NA, Sutradhar P. Karydakis procedure for sacrococcygeal pilonidal sinus disease: Our experience. Indian Journal of Plastic Surgery. 2014 Sep; 47(03):402-6.

7. Michalopoulos N, Sapalidis K, Laskou S, Triantafyllou E, Raptou G, Kesisoglou I. Squamous cell carcinoma arising from chronic sacrococcygeal pilonidal disease: A case report. World journal of surgical oncology. 2017 Dec; 15(1):65.

8. Fazeli MS, Adel MG, Lebaschi AH. Comparison of outcomes in Z-plasty and delayed healing by secondary intention of the wound after excision of the sacral pilonidal sinus: Results of a randomized, clinical trial. Diseases of the colon \& rectum. 2006 Dec $1 ; 49(12): 1831-6$

9. Karapolat B, Büyükakıncak S, Kurnaz E, Küçüktülü Ü. Comparison of limberg flap and oval flap techniques in sacrococcygeal pilonidal sinus disease surgery. Turkish journal of surgery. 2018; 34(4):311.

10. Sadiq F, Azeem M. Karydakis technique for pilonidal sinus: Revisited. PJMHS. 2017:11(2):742-44

.11. De Robles MS, Seyfi D, Zahid A, Young CJ. Karydakis procedure can be effectively performed in the lateral position. ANZ journal of surgery. 2019 Jan; 89(1-2):E104. 


\begin{tabular}{l} 
AUTHORSHIP AND CONTRIBUTION DECLARATION \\
\begin{tabular}{|l|l|l|}
\hline Sr. \# & Author(s) Full Name & \multicolumn{1}{|c}{ Contribution to the paper } \\
\hline 1 & Durr-I-Chaman & Main author. \\
2 & Tayyaba Fatima & Co-researcher. \\
3 & Muhammad Akram & Co-researcher. \\
4 & Bashir Ahmed & Co-researcher. \\
5 & Sajid Rehman & Co-researcher. \\
6 & M. Sajid Sheikh & Supervisor.
\end{tabular} \\
\hline
\end{tabular}

